\section{$\underset{\substack{\text { hommes } \\ \text { \& migrations }}}{ }$}

\section{Hommes \& migrations}

Revue française de référence sur les dynamiques

migratoires

1282 | 2009

Santé et droits des étrangers : réalités et enjeux

\title{
Amazigh et Kamel, dignes fils de leurs pères
}

\section{François Bensignor}

\section{CpenEdition \\ Journals}

\section{Édition électronique}

URL : http://journals.openedition.org/hommesmigrations/473

DOI : 10.4000/hommesmigrations.473

ISSN : 2262-3353

\section{Éditeur}

Musée national de l'histoire de l'immigration

\section{Édition imprimée}

Date de publication : 1 novembre 2009

Pagination : 186-193

ISSN : 1142-852X

\section{Référence électronique}

François Bensignor, "Amazigh et Kamel, dignes fils de leurs pères », Hommes \& migrations [En ligne], 1282 | 2009, mis en ligne le 29 mai 2013, consulté le 22 septembre 2020. URL : http:// journals.openedition.org/hommesmigrations/473 ; DOI : https://doi.org/10.4000/hommesmigrations. 473

Ce document a été généré automatiquement le 22 septembre 2020.

Tous droits réservés 


\title{
Amazigh et Kamel, dignes fils de leurs pères
}

\author{
François Bensignor
}

\section{Amazigh Kateb}

1 Après quinze ans d'aventure avec les musiciens de son groupe, Gnawa Diffusion, Amazigh Kateb a souhaité prendre de la distance. Dorénavant, il assume seul la direction de sa carrière. S'il n'a rien perdu de l'esprit contestataire, facétieux et festif qui s'était imposé avec le groupe, la maturité de son inspiration s'affirme au détour de nouvelles préoccupations et de nouveaux questionnements personnels dans Marchez noir. Ce premier album sous son nom continue de dénoncer sur des rythmes stimulants, d'appuyer où ça fait mal, de chercher la vision à la limite du délire. Mais s'y dessine aussi la quête d'une continuité par rapport à l'œuvre de son père, dont pour la première fois, à l'occasion des vingt ans de sa mort, il s'autorise à mettre en musique des poèmes.

2 "Bonjour", qu'il a puisé dans ses œuvres de jeunesse, figure une ouverture magnifique. Habité par la lucidité rimbaldienne du désespoir qui transcende les mots, ce texte, troussé dans une mélodie chaâbi qui lui va comme un gant, contient toute la force d'un esprit libre, nouvellement né au monde des lettres. Délibérément, Amazigh choisit de projeter une image de son père en jeune poète à peine sorti de l'enfance, que le destin vient de plonger dans les pires affres de l'angoisse. Lorsqu'il écrit ce texte, Yacine est encore un adolescent, idéaliste, féru de culture française. Il croit en l'avenir, mais vient de faire l'expérience de l'horreur et traduit les tourments qui l'assaillent avec une simplicité poignante. Le contexte historique dans lequel ce poème a été écrit fait froid dans le dos.

3 Nous sommes le 8 mai 1945. L'Algérie fête la libération de la France dans la joie. La journée a été décrétée fériée. À Sétif, des Algériens qui, ayant combattu pour la France, pensent pouvoir obtenir un meilleur statut que celui qui leur est imposé par les autorités coloniales, en profitent pour protester. Ils réclament un traitement égalitaire 
et bientôt dans le cortège s'élève la voix des nationalistes. L'ambiance est explosive. Soudain, c'est la mêlée. Des coups sont échangés avec les policiers qui réagissent avec violence et la situation dégénère en émeute. Des Français sont pris à partie, certains y laissent leur vie. Une répression sanglante va découler de ces événements, "les massacres de Sétif" de triste mémoire, l'un des épisodes les plus noirs de l'histoire franco-algérienne. La campagne de représailles lancée par l'armée coloniale se prolonge durant plusieurs semaines, faisant entre 8000 et 45000 morts, selon les sources. Kateb Yacine, alors âgé de 15 ans, avait rejoint des camarades dans le défilé. Voyant la violence se déchaîner, il était reparti dans son village. Mais on l'a vu et dénoncé. Les gendarmes viennent l'arrêter et le jettent en prison. Pendant ses quatre mois d'enfermement, le jeune Yacine est témoin d'exécutions sommaires et de la sauvagerie des soldats. La femme qui lui enseigne le français intercède en sa faveur et il a la chance d'en réchapper. "Bonjour" jaillit après cet épisode douloureux. Il y exprime son désarroi autant que la perte de ses illusions.

"Quand il évoquait cette période, mon père disait que c'était en prison qu'il avait découvert l'Algérie dont il parle dans ses livres, explique Amazigh. En tant que fils d'avocat, il allait à l'école française, avait des amis italiens, maltais, portugais. Il aimait beaucoup la langue française. Ses amis algériens étaient pour la plupart issus d'une classe de lettrés. Kateb signifie d'ailleurs 'le lettré.' Yacine faisait partie d'une famille protégée. C'est dans la manifestation de Sétif qu'il a vu ses premiers morts. Et ensuite, en prison, il a compris que l'Algérie était dans un état de misère extrême."

5 "Mon père a écrit son premier recueil de poésies, Soliloques, entre 14 et 15 ans, poursuit Amazigh. Un an après, il avait réussi à en taper le manuscrit à la machine. Il le portait avec lui un matin, dans un bar d'Annaba [appelée 'Bône' à l'époque]. Il était amoureux et venait de passer une nuit blanche. Des ouvriers commençaient leur journée au vin blanc. Dans ce bar, un homme lui demande : 'Qu'est-ce que tu as avec toi de si précieux.' Mon père explique qu'il écrit de la poésie, mais qu'il est difficile de se faire éditer. Et l'homme lui répond: 'Eh bien, je suis imprimeur. Ma société est en faillite, mais il me reste encore de l'encre et du papier.' C'est ainsi que les cent premiers exemplaires de Soliloques ont été imprimés en 1946 à l'imprimerie du Réveil bônois. Mon père est allé déposer des livres un peu partout, dans les bars, les épiceries, chez les coiffeurs. La plupart des gens qui ont acheté les premiers exemplaires de Soliloques étaient analphabètes. Ils le faisaient par solidarité: c'était si rare un jeune poète algérien écrivant en français !..."

6 Autre texte de Yacine, "Africain" est aussi en musique dans l'album d'Amazigh, deuxième fleur offerte à l'occasion du vingtième anniversaire de la mort de son père, le 28 octobre 1989. "C'est le seul jour férié que je m'accorde", glisse-t-il. On mesure là l'amour que le fils a toujours voué au génie qui l'a enfanté et dont il demeure l'un des admirateurs les plus fervents. En fait, c'est à l'occasion d'une commande qui lui avait été passée par la ville de Roubaix en 2006 qu'Amazigh s'est immergé dans l'œuvre de Yacine. "J'ai vécu toute l'époque où mon père montait ses pièces, explique-t-il. Je me rappelle très bien des répétitions, et notamment des mélodies et des musiques. Comme ses pièces de théâtre étaient très évolutives, il n'en existe pas de version définitivement fixée. Même par l'écriture! En reprenant les textes qu'il donnait aux comédiens pour apprendre leurs rôles, je me suis aperçu qu'ils variaient avec le temps. C'était d'ailleurs un casse-tête pour les acteurs qui devaient sans cesse répéter les nouvelles versions de leurs répliques. J'estimais qu'il était de mon devoir de préserver cette matière." 
7 Le projet de monter la pièce Mohamed, prends ta valise! dans le cadre du Festival de l'Amitié à Roubaix, avec quatre comédiens et un groupe de musiciens, ne verra jamais le jour sur scène... "Il a été censuré par les commanditaires eux-mêmes, parce qu'en France la question coloniale n'est apparemment pas encore digérée", explique Amazigh, désabusé. "Mais le fait de me remettre dans l'écriture de mon père m'a débridé. Ce travail d'archéologue m'a remué les méninges et les tripes. Deux fois, en jouant pendant les répétitions, mon émotion a explosé en crise de larmes. Je n'étais pas triste. J'avais gardé en moi des nœuds. Pendant des années, nourri par l'euphorie de Gnawa Diffusion, j'avais mis mon deuil de côté. Il était avec moi sans m'encombrer et il est remonté en travaillant sur Yacine. Après cette expérience, il est devenu clair dans ma tête que j'avais un nouveau chemin à parcourir, habité par cette impulsion. Je me suis réapproprié quelque chose qui m'habite depuis des années et qui s'est déclaré."

8 "Ce que je trouve de plus beau dans la vie de mon père telle que je la vois, c'est son intégrité aussi bien par rapport à la francophonie que par rapport à l'Algérie. À l'époque où le pays n'était pas encore indépendant, il avait des positions très nettes vis-à-vis du FLN. Il s'est toujours démarqué, n'a jamais pris une carte dans aucun parti, même s'il a aidé à appeler à certaines mobilisations. Mon père a été écrivain public dans les rues de Paris. Par cette seule position, il a coordonné des quartiers entiers d'émigration. L'écrivain public est le confident de tout le monde. C'est aussi ce qui a fait de lui le personnage qu'il est devenu: pas seulement un écrivain mais incarnant la conscience d'un peuple. Beaucoup de gens se rappellent de lui. À Ménilmontant, je croise par hasard un nombre impressionnant de personnes qui me disent qu'elles ont connu mon père à telle époque, bu un verre avec lui, qu'elles l'ont vu s'énerver ou se saouler..."

Lorsqu'on lui demande comment il se situe par rapport à l'époque qu'a vécue son père, Amazigh affirme : "Je me sens enfant et héritier d'une histoire. La génération de mes parents a vécu la mutation entre le statut de colonisé et celui de peuple 'indépendant' - avec toute la relativité que l'on peut appliquer à ce terme. Et je remercie mon père de m'avoir transmis ce qu'il a pu comprendre à travers son travail et les difficultés qui étaient inhérentes à cette époque. Il ne m'a pas transmis ses difficultés, et c'est heureux, mais j'ai compris des choses grâce à lui, sans avoir à en faire l'expérience. Par exemple, je sais que le cheminement qui l'a mené de la poésie au roman, puis au journalisme et jusqu'à l'oralité théâtrale visait à toucher le plus grand nombre possible de gens. Cette démarche a beaucoup compté dans ma vie. J'ai toujours eu en moi cette volonté de faire quelque chose pour les gens et pour le plus grand nombre possible, sans qu'il y ait d'obstacle de 'lecture'. Autant dans la peinture que dans l'écriture, il peut y avoir une abstraction que le profane, celui qui n'est pas formé, ne va pas comprendre. Le choix de mes modes d'expression - la musique, les mots exprimés verbalement-a été influencé par l'expérience de mon père, qui a duré soixante ans. Pour moi, c'est un véritable héritage."

\section{Kamel El Harrachi}

10 Au printemps 2009, Kamel El Harrachi a publié son premier véritable album, Ghana Fenou ("il a chanté son art"), en hommage à son père, Dahmane, disparu le 31 août 1980. L'auteur de "Ya Rayah" - devenu un chef d'œuvre international par la grâce de Rachid Taha - laissait un répertoire de près de 750 chansons. Sur les accords mélancoliques du chaâbi algérois, elles content les heurs et malheurs des émigrés économiques, dont il faisait partie. Vivant loin de son père, qui toujours à ses yeux figure comme une icône, Kamel s'est imprégné de sa musique. Depuis ses 18 ans, il fait revivre avec une authenticité unique l'œuvre d'El Harrachi. 
11 “Malheureusement, je n'ai pas bien connu mon père: je n'avais que 7 ans quand il nous a quitté. Et mon plus grand regret est de n'avoir pas pu jouer sur scène avec lui", dit Kamel. Il est l'aîné des quatre enfants d'Abderrahmane Amrani, qui avait hérité son nom de scène du quartier d'El-Harrach, où il avait grandi à Alger. Dès 1948, Dahmane s'est exilé en France. D'abord musicien amateur, ses premiers enregistrements remontent à 1956 et sa réputation de professionnel se construit dans les cafés et cabarets maghrébins, où les ouvriers étanchent au bar la nostalgie du pays. Roubaix, Lille, Nice, Marseille, Toulouse, Bordeaux et bien sûr Paris, où il passe la plus grande partie de sa vie, ont gardé le souvenir de ce chanteur élégant à la voix rocailleuse, aux rimes assurées et qui aimait la vie. Au point que la bouteille était devenue pour lui une compagne insidieuse. Comme dans le blues, l'alcool s'invite parfois dans les paroles de son chaâbi: "Ach eddani nkhatou" (“Qu'est ce qui m'a pris de le fréquenter ?"), “Rabbi blani bettassa" (“Dieu m'a doté de la tare de l'alcool”). Mais pour son fils aîné, né en 1973 alors que la carrière du père est déjà installée, c'est la musique qui compte. Elle tisse ce lien étroit qui l'unit à sa figure absente.

"Je n'ai même pas le souvenir de la façon dont j'ai appris à jouer", explique Kamel. "Je mettais des disques de mon papa à la maison, j'accordais mon instrument sur sa musique et je jouais. J'avais une mandoline et le mandole de mon père. Il en avait deux, un qui le suivait partout, l'autre qu'il laissait toujours à la maison, accordé dans sa sacoche. Quand je rentrais le soir, j'aimais prendre son instrument et jouer. C'est ainsi que j'ai appris. Je n'ai jamais eu de professeur. Je suivais la mélodie des disques. Plus tard, je me suis perfectionné en jouant avec des amis, en regardant d'autres artistes et en écoutant d'autres musiques."

Né en Algérie, Kamel n'a vécu que deux ou trois années à Lyon auprès de son père, qui avait fait venir en France sa femme et ses enfants. Mais vie de famille et vie d'artiste ne font pas forcément bon ménage... La mère préfère élever ses enfants hors du tumulte des concerts. "La musique a toujours tenu la première place dans notre vie", explique Kamel. "Mais la vie d'artiste n'est jamais très facile... D'ailleurs mon père ne voulait pas que je sois chanteur. Il souhaitait que je fasse des études pour pouvoir accéder à un autre métier. Il avait tellement souffert et galéré dans ce milieu de musiciens qu'il ne voulait pas que son fils passe par ce qu'il avait vécu. Je ne le comprenais pas quand j'étais gamin, mais aujourd'hui je sais pourquoi il disait ça."

Quoi qu'ait pu exprimer le père absent, c'est la passion de la musique qui prend le dessus. Kamel le comprend dès ce jour de 1985 où il a l'occasion d'interpréter deux chansons de son père en public, lors d'un concours de chaâbi. À 12 ans, il voit déjà son destin tracé. Il décroche la troisième place, mais est bien décidé à travailler autant qu'il le faudra pour acquérir la maîtrise en cette matière. "J'aime le chaâbi", insiste Kamel. "C'est un style de chanson qui fait sens. Beaucoup de gens ne comprennent pas. Ils pensent que je devrais faire $d u$ rap ou $d u$ rock... Ce sont des musiques que j'écoute, mais ce qui me plaît vraiment, c'est le blues algérien: le chaâbi. Certaines chansons parlent d'amour ou d'amitié, d'autres de trahison ou d'émigration, toutes sortes de problèmes qui touchent la société. Le chaâbi est basé sur le texte. Chaque chanson doit avoir du sens, être ressentie par l'auditeur comme une part de son vécu. Lorsque j'étais en Algérie, récemment, un vieil homme m'a dit : 'Tu sais mon fils, lorsque je me sentais un peu malade à l'époque, je n'allais pas à la pharmacie, je rentrais à la maison et j'écoutais un disque de ton père. C'était comme si j'avais été voir un médecin."”

En 1995, Kamel, qui a déjà son public, est un des fers de lance du renouveau chaâbi en Algérie, lorsque déferle la folie intégriste. Avec sa famille, ils n'ont qu'une solution, 
suivre les traces du père en s'exilant en France. Une nouvelle vie, de nouveaux circuits sont à construire. Kamel prend son temps : il veut faire les choses bien, se faire accepter non seulement comme le fils, mais comme le successeur de Dahmane. Or, aujourd'hui, Kamel est mûr. Reconnu par sa communauté, on le demande aussi sur les scènes du monde, jusqu'en Australie. L'album Ghana Fenou est placé parmi les vingt premiers du classement des radios européennes de musiques du monde, World Music Charts Europe, pour 2009.

“'Ghana Fenou', la chanson titre de l'album, est une de mes compositions où je rends hommage à mon père, explique Kamel. Sur le disque, je reprends plusieurs de ses chansons écrites dans les années soixante, en leur donnant une couleur d'aujourd'hui. Beaucoup de jeunes ne connaissent pas ces chansons et j'ai envie de leur donner une seconde vie. On me demande aussi de réinterpréter des titres qui n'existent plus sur le marché. Certains morceaux de mon père sortis en 45 tours sont aujourd'hui introuvables. Si personne ne les reprend, ils disparaitront. Ma version leur permettra de vivre encore quelque temps."

\section{BIBLIOGRAPHIE}

Marchez noir - Amazigh Kateb (Iris Music / Harmonia Mundi, 2009)

Ghana Fenou - Kamel El Harrachi (Turn Again Music / Mosaic Music Distribution, 2009)

\section{NOTES}

1. Poème édité dans L'œuvre en fragments (Éditions Sindbad, 1986), dont voici la première strophe :

"Bonjour ma vie

Et vous mes désespoirs

Me re-voici au fossé

Où naquit ma misère

Voici le coin de boue

Où dormait mon front fier

Voici ma vie à moi

Rassemblée en poussière"

\section{RÉSUMÉS}

À l'occasion de l'exposition Générations, un siècle d'histoire culturelle des Maghrébins en France, nous avons souhaité donner la parole aux enfants de deux icônes populaires qui ont marqué 
celle-ci de leur sceau. Amazigh Kateb, fils de Kateb Yacine, et Kamel El Harrachi, fils de Dahmane El Harrachi, tentent, chacun à leur manière, de perpétuer un nom tout en faisant valoir l'originalité de leurs prénoms. Issus de la même génération - le premier est né en 1972, le second en 1973 - tous deux inscrivent leurs carrières respectives dans la continuité de celles des personnalités hors norme qui les ont enfantés. En 2009, l'un et l'autre ont publié leur premier album : Marchez noir pour Amazigh, Ghana Fenou pour Kamel. Chacun y rend hommage à son père. Comment vivent-ils cette filiation? À cette question, ils apportent leurs réponses personnelles. 